\title{
Estudio del comportamiento dinámico en edificaciones irregulares considerando interacción suelo estructura
}

\author{
Study of dynamic behavior in irregular buildings considering \\ interaction soil structure
}

\author{
María Arellano \\ mariaeaq1094@gmail.com \\ Johana Cavallin \\ Universidad de Carabobo, Venezuela
}

\section{RESUMEN}

El presente trabajo es el resultado obtenido del estudio del comportamiento dinámico en edificaciones irregulares como: distribución irregular de la masa en uno de los pisos contiguos, variación en la geometría vertical, edificaciones apoyadas en pendiente del terreno, esbeltez excesiva y sistemas no ortogonales, considerando interacción suelo-estructura. Se establecieron cuatro modelos estructurales por irregularidad, los cuales están conformados por un modelo regular (MB) y tres modelos irregulares M2, M3 y M4 respectivamente. Las fundaciones fueron planteadas como base rígida al inicio del análisis para posteriormente ser estudiadas bajo el efecto de interacción suelo estructura, dando lugar a fundaciones flexibles. El análisis se hizo empleando el Método de Superposición Modal con Tres Grados de Libertad, tomando en cuenta los parámetros establecidos en la Norma COVENIN "Edificaciones Sismoresistentes" (1756:2001), para las Formas Espectrales S1, S2 y S3. Los resultados permitieron realizar comparaciones en las formas de vibración, las derivas de piso y el cortante basal.

Palabras clave: Interacción sueloestructura; forma espectral; corte basal; derivas

\begin{abstract}
The present work of degree is the result obtained from the study of the dynamic behavior in irregular buildings, as irregular distribution of the mass in one of the contiguous floors, variation in the vertical geometry, buildings supported by slope of the ground, excessive slenderness and non-orthogonal systems, considering soil-structure interaction. Four structural models were established by irregularity, which are conformed by a regular model (MB) and three irregular models M2, M3 and M4 respectively. The foundations were raised as a rigid basis at the beginning of the analysis, and later they were studied under the effect of soil structure interaction, giving rise to flexible foundations. The analysis was made using the Modal Superposition Method with Three Degrees of Freedom, taking into account the parameters established in the COVENIN Standard "Sismoresistentes Buildings" (1756: 2001), for the Spectral Forms S1, S2 and S3. The results obtained allowed us to make comparisons in the forms of vibration, the floor drifts and the basal shear.
\end{abstract}

Key words: Soil-Structure Interaction; spectral form; basal cut; drifts 


\section{INTRODUCCIÓN}

Uno de los eventos naturales que causan el colapso de las edificaciones son los sismos los cuales son inducidos por movimientos inesperados provenientes de la interacción entre las placas tectónicas de la corteza terrestre (Yamin et al., 2003; Alfaro, et al., 2013). A su vez, estos movimientos generan lo que se conoce como vibraciones sísmicas (Caicedo, 2014) manifestadas en el suelo como fuerzas transmitidas en varias direcciones. Desde el punto de vista del diseño estructural en la ingeniería civil (Merritt, 1992) si estas fuerzas no son consideradas pueden llegar a causar pérdidas tanto económicas como vidas humanas (Hernández-Castañeda y Mendoza-Escobedo, 2006).

Debido a la existencia de regiones sísmicas en varias partes el mundo (Ordaz, et al., 2015) se debe conocer las propiedades dinámicas del suelo que permitan comprender cómo se transmiten los distintos movimientos de este a las cimentaciones $\mathrm{y}$, por consiguiente, a la estructura, junto a los efectos de interacción que se generan (Cruz et al., 2004; Nuria y González, 1995; Rucks et al., 2004). Uno de los aspectos que suele pasarse por alto, y es de gran importancia al momento de diseñar una edificación, consiste en la interacción suelo estructura donde el terreno sufre ciertas modificaciones, las cuales se ven reflejadas en su base. Aunado a esto se suman otros factores que influyen en el comportamiento dinámico de las edificaciones cuando en estas existen irregularidades geométricas determinadas por la ubicación o la configuración de los elementos estructurales que las integran (Vielma y Mulder, 2018; Vanegas y Giraldo, 2017).
En la actualidad se cuenta con importantes investigaciones y estudios enfocados en resaltar las causas principales del colapso de edificaciones en presencia de eventos sísmicos, los cuales se basan en las catástrofes ocurridas, a lo largo de los años, en regiones altamente sísmicas cobrándose la vida de muchas personas (Blanco, 2012; Alba y Homero , 2017; Guevara y Sánchez, 2005). Dichas causas logran demostrar que una falta de consideración entre la geometría de la edificación y las condiciones generales del suelo conducen a un mal diseño estructural $\mathrm{y}$, por lo tanto, se obtiene un mal comportamiento de los elementos que la rigen.

A pesar de las causas expuestas, profesionales en el área de la construcción suelen pasar por alto estas referencias, muchas veces por falta de conocimiento acerca del tema (Bairral, 2005; Tejada; 2005). Para efectos de este estudio se tendrán presentes algunas de estas consideraciones, lo que conlleva al planteamiento de modelos con distintas irregularidades estructurales, las cuales dependen del grado de interacción arrojado por la respuesta sísmica de la edificación, además de las características de un suelo blando previamente establecido. Cada tipo de irregularidad estará aplicada a modelos estructurales detallados según el parámetro que las define.

Las irregularidades establecidas para la ejecución de este trabajo fueron escogidas por variables inducidas según problemas comunes que se pueden llegar a presentar, tales como la construcción de edificaciones que tiendan a sufrir de un riesgo torsional elevado (Lee, et al., 2007). Se plantearon modelos con pendientes del 
terreno elevadas, al igual que edificaciones con líneas resistentes no ortogonales en dirección del eje "Y". Así mismo, se estudiarán edificaciones con gran esbeltez, conformadas por aquellas columnas que puedan llegar a experimentar deformaciones por una falta de estabilidad. Lo que da lugar a que la misma pueda llegar a adoptar problemas de volcamiento y elevadas transmisiones de carga a las cimentaciones. Cabe destacar que esto conlleva a tener presente ciertos procedimientos para que su diseño sea factible $y$ confiable. Otras de las irregularidades son aquellas con distribución irregular de masas en la elevación y las de variabilidad en la geometría vertical del diseño estructural, cuyo diseño puede traer como consecuencia concentraciones de demandas inelásticas indeseables en puntos desfavorables de la edificación.

Basados en la ocurrencia de estas posibles problemáticas se propone un estudio del comportamiento dinámico en edificaciones irregulares como, distribución irregular de la masa en uno de los pisos contiguos, variación en la geo-metría vertical, edificaciones apoyadas en pendiente del terreno, esbeltez excesiva y sistemas no ortogonales, considerando interacción suelo-estructura. Se establecieron cuatro modelos estructu-rales por irregularidad, los cuales están conformados por un modelo regular (MB) y tres modelos irregulares M2, M3 y M4. (Arellano, et al., 2018).

\section{MATERIALES Y MÉTODOS}

\section{Fase 1. Selección de los modelos a evaluar}

Esta etapa de la investigación tiene como finalidad la selección de los modelos estructurales a evaluar en los cuales se pueda evidenciar las irregularidades en la geometría en planta y elevación. Esto modelos fueron seleccionados tomando en cuenta parámetros básicos para su diseño como el número de líneas resistente, uso, tipo de material, zonificación sísmica y lo establecido en la Norma COVENIN (17562001) (Arellano, et al., 2018; Arteaga et al., 2014).

Inicialmente se plantea una estructura regular (MB), la cual no presenta discontinuidad en su geometría. Seguidamente, se definieron los modelos irregulares (M2, M3 y M4) en base a la Distribución Irregular de masa de uno de los pisos contiguos, variaciones de la geometría del sistema estructural, esbeltez excesiva, sistemas no ortogonales y edificaciones apoyadas en pendientes. El MB consta de cuatro (4) ejes sismos resistente tanto en " $\mathrm{X}$ " como en "Y" con separación de 4,5 m. La estructura está conformada por diez (10) pisos de altura con entrepisos de tres (3) $\mathrm{m}$.

Para la irregularidad, la Distribución Irregular de la masa en uno de los pisos continuos, se definieron tres (3) modelos estructurales, los cuales constan igualmente de cuatro (4) ejes sismos resistentes tanto en " $\mathrm{X}$ " como en "Y" con separación de 4,5 m y diez (10) pisos en total, con entrepisos de tres (3) $\mathrm{m}$ de altura. El MB lo conforma una estructura regular, en el cual no se aumentará la masa ni sufrirá ningún tipo de cambio. No obstante, para el M2 el aumento de la masa 
se define en el nivel 6, como 1,3 veces la masa del piso contiguo. Al igual que para el M3 y M4, cuyo aumento de la masa será en el nivel 8 y el nivel 10, respectivamente.

En cuanto a la Irregularidad de Variaciones de la Geométrica del Sistema Estructural, se definieron tres (3) casos de estudios conformado por tres (3) modelos estructurales conformados por cuatro (4) ejes sismos resistentes tanto en " $\mathrm{X}$ " como en "Y", con separación de 4,5 m, de 10 pisos y con entrepisos de tres (3) $\mathrm{m}$ de altura. Se reduce el número de ejes sismos resistentes respecto al modelo base desde el nivel 10 hasta en nivel 8. Por otra parte, para la Irregularidad con Esbeltez Excesiva, se plantearon cuatro (4) modelos caracterizados por la relación H/B con variación entre dos (2) para el MB, tres (3) para el M2, 4,29 para el M3 y 4,62 para el M4 respectivamente. En este caso, los ejes sismoresistentes varían según la relación H/B correspondiente a cada modelo.

En el caso de la Irregularidad con Sistemas No Ortogonales, se definieron tres (3) casos de estudios integrados por tres (3) modelos estructurales conformado por diez (10) pisos, con entrepisos de tres (3) m de altura. Partiendo del MB antes planteado, el resto de los modelos presentan variaciones ortogonales de las líneas sismo resistentes en dirección " $\mathrm{Y}$ ", donde el M2 varía con un ángulo $\beta$ de $5^{\circ}$, el M3 con un $\beta$ de $10^{\circ}$ y el M4 con un $\beta$ de $15^{\circ}$.

En la Irregularidad con Edificaciones apoyadas en pendientes, se definieron tres (3) modelos estructurales, los cuales constan de cuatro (4) ejes sismos resistentes tanto en " $\mathrm{X}$ " como en " $\mathrm{Y}$ " con separación de 4,5 m, 10 pisos en total y con entrepisos de $3 \mathrm{~m}$ de altura. Partiendo del MB regular, se define el primer modelo de esta irregularidad, denominado M2 con una inclinación de la pendiente del terreno $\beta 5^{\circ}$, el M3 presenta una inclinación en la pendiente del terreno de $\beta 10^{\circ}$ y $\mathrm{M} 4$ contara con una inclinación de la pendiente del terreno de $\beta 15^{\circ}$.

Para la definición geométrica de los modelos irregulares con Edificaciones apoyadas en pendiente, fue necesario efectuar algunos cálculos matemáticos que permitieran conocer las longitudes de las columnas apoyadas sobre la pendiente. Partiendo de un parámetro fijo, en este caso el ángulo de inclinación $\beta$ que debe tener la pendiente planteada inicialmente, y la cual varía según cada modelo definido, así como la altura de la columna D fijada en tres (3) m para evitar efectos de columna corta y posibles fallas en el sistema estructural. Se pudo entonces encontrar el resto de las distancias y valores requeridos aplicando una relación de triángulos.

\section{Fase 2. Análisis y Modelado Estructural}

El diseño de los 52 modelos estructurales con bases rígidas se lleva a cabo considerando sistemas de pórticos en ambas direcciones conformados por columnas, vigas y entrepisos compuestos por losas nervadas, armadas en dos direcciones. El análisis se realiza mediante el Software de cálculo estructural ETABS [21], donde se estiman las cargas gravitaciones como las cargas sísmicas. Además, se evalúa el comportamiento de cada modelo considerando las verificaciones de parámetros como el cortante basal, derivas, cuantías y 6/5 vigas/columnas según las consideraciones estipuladas en la Norma COVENIN (17562001). 
El procedimiento es descrito para un (1) sólo modelo, en este caso el modelo base MB de las irregularidades con forma espectral 1 (S1), debido a que los pasos a seguir son los mismos para todos los demás. Sin embargo, para la irregularidad distribución irregular de la masa en uno de los pisos continuos, se describe el procedimiento empleado en el programa para la asignación de la masa en el piso correspondiente.

De acuerdo a la descripción anterior sobre la geometría en planta del $\mathrm{MB}$, se sabe que este posee 4 líneas resistentes tanto en dirección " $\mathrm{X}$ " como en dirección "Y". Cada una de ellas guarda una distancia de 4,5 m de separación. El primer paso consiste en definir la grilla sobre la cual se levantará el modelo estructural de acuerdo a los 10 niveles.

\section{Fase 3. Interacción Suelo-Estructura}

Luego de haber aplicado el Análisis Dinámico Modal a los 52 modelos estructures con base rígida, se procede a introducir la Interacción Suelo-Estructura. Generando de esta forma un total de 52 modelos adicionales, modelados con base flexible y cimentaciones profundas $\mathrm{o}$ pilotes que trabajen por punta.

\section{RESULTADOS}

Luego de realizar el respectivo análisis de los modelos estructurales en el programa de diseño ETABS, se obtuvieron los datos resultantes de los mismos en conjunto con el análisis de interacción suelo estructura. Estos serán presentados a continuación, mediante gráficos que comprenden el comportamiento de las derivas, el cortante basal y las formas modales según la irregularidad, la forma espectral y la dirección. Sin embargo, en cuanto a los resultados obtenidos según la dirección de estudio en $\mathrm{Y}$, estos serán excluidos de esta sección, siempre y cuando mantengan un comportamiento semejante respecto a la dirección $\mathrm{X}$ (Arellano et al., 2018). En este sentido, se presentan las siguientes observaciones:

\section{Irregularidad con Distribución de Masas en la Altura}

- En cuanto a los resultados obtenidos en la comparación del cortante basal, se pudo apreciar que mientras la estructura tendía a aumentar su masa en los pisos superiores en suelos poco flexible el cortante basal aumentaba considerablemente para los modelos planteados con interacción suelo estructura respecto a los modelos de base rígida, siendo este aumento de aproximadamente de 33\%. Esto indica que para este tipo de edificación sus elementos estructurales presentan una demanda alta en cuanto a sus dimensiones, lo cual conlleva al aumento de sus dimensiones para su correcto funcionamiento.

- Se observa que a medida que la estructura aumenta su masa en el nivel más elevado y se encuentra cimentada sobre suelos muy flexible, los desplazamientos para el caso de los modelos con interacción suelo estructura, superan el límite establecido en la norma. Y a su vez también tienden a ser más elevados que los modelos con base rígida.

- Respecto a las formas modales, se observa que tanto para las edificaciones con bases rígidas y bases flexibles, el desplazamiento aumentaba 
hacia los niveles superiores de la estructura. Esto se traduce en un aumento de las tensiones en las columnas inferiores.

\section{Irregularidad de Edificaciones Apoyadas en pendiente}

- En este caso, se presencia que los modelos con ángulo de inclinación de pendiente de 10 grados, fueron los que presentaron un aumento del corte basal más elevado, para las tres formas espectrales planteadas respecto al resto de los modelos. Cabe destacar que estos modelos al encontrarse apoyados en suelos muy rígidos el aumento del corte considerando interacción suelo estructura, arrojo una variación del $32,55 \%$ respecto al resto de los modelos.

- Las derivas en esta irregularidad tienden a aumentar en los modelos con base flexible en dirección $\mathrm{Y}$ para modelos con interacción suelo estructura a medida que la estructura se apoya en un terreno con mayor inclinación, dando origen a desplazamientos de gran magnitud en los primeros pisos de la edificación. A pesar que esta tendencia se mantiene para la mayoría de los modelos estructurales, se logró excluir un modelo en particular en cual los desplazamientos obtenidos fueron muy bajos, gracias a que el mismo es un modelo tan irregular que a la hora de cumplir con otros chequeos planteados en la Norma COVENIN, fue necesario aumentar la dimisión de sus elementos estructurales, dando origen a una edificación muy robusta.

- En cuanto a las formas modales obtenidas para este tipo de irregula- ridad, se observó que para los modelos de base rígida para todas las formas espectrales, estando en presencia de un periodo de vibración corto, esta describe desplazamientos despreciables.

\section{Irregularidad Gran Esbeltez}

- En las edificaciones con gran esbeltez, se evidencia que la tendencia del incremento de los desplazamientos mayores se obtuvo para los modelos que se apoyan en suelos más flexibles sin importar que tan irregular fuese. También, se pudieron apreciar algunos modelos en los cuales el valor de las derivas no fue tan considerable, este caso el diseño se ve gobernado por las cargas gravitacionales.

- En cuanto a los resultados obtenidos en el cortante basal el modelo más esbelto arrojó los mayores valores de corte basal respecto al resto de los modelos, esto se debe a que mientras más esbelta es la estructura, la misma va exigir el aumento de sus elementos estructurales lo que hará a su vez que su peso sísmico aumente. Se pudo notar, además, la similitud de los cortes obtenidos tanto para los modelos con base rígida como los modelos con base flexible, sin embargo, a pesar de que la diferencia es muy poca el corte basal predomina para edificación sin interacción suelo estructura.

- Para el modelo número 2 (relación H/B: 2) arrojó un periodo de excitación mayor en los modelos con base flexible, lo cual generó la amplificación de los desplazamientos significativamente. Además, se pudo notar como para los modelos apoyados en un 
suelo poco flexible, el periodo de vibración tiende a ser mucho más amplio para los modelos con interacción suelo estructura respecto a los modelos de base rígida, por lo que la estructura adquiere desplazamientos muy elevados hacia los niveles superiores, originando un aumento de las tensiones en las columnas de los niveles inferiores $\mathrm{y}$ por ende su colapso.

\section{Irregularidad con Variación de la Geometría Vertical}

- Tras comparar las derivas de los modelos irregulares con variación de la geometría vertical pudimos observar como a medida que la estructura disminuye sus líneas sismoresistente en los niveles 10,9 y 8 esta tiende a describir un amento discreto de los desplazamientos al ser evaluados con interacción suelo estructura. Siendo el M2 con forma espectral S3 el que presenta una mayor disminución de los desplazamientos hacia los niveles superiores en casi un $90 \%$ de toda la edificación. Esto se debe porque cuando se está en presencia en este tipo de suelos, las ondas sísmicas tienden a amplificarse a través del estrato, generando periodos de vibración muchos más largos, lo que da origen a mayores desplazamiento a los largo de la estructura.

- La tendencia arrojada por el cortante basal en los modelos de este tipo de irregularidad, señala un aumento considerable en los modelos apoyados sobre suelos rígidos, tras ser sometidos con el estudio de interacción suelo estructura. Dicha diferencia del incremento se refleja en gran porcentaje a medida que la estructura disminuye sus líneas resistente en los niveles superiores.

- En conclusión, respecto al comportamiento de las formas modales, no cabe duda que tanto para los modelos con base rígida como para los modelos de base flexible, los desplazamientos aumentan progresi-vamente hacia los niveles superiores de la estructura. Desde otro punto de vista, se tiene que el movimiento descrito por las masas de la edificación son los referidos al modo de vibración 1 , donde se tiende a trasladar por completo la masa hacia un solo sentido, aun cuando la estructura es irregular.

\section{Irregularidad con Sistemas No Ortogonales}

- Una vez estudiado el comportamiento de las derivas de piso, los desplazamientos en estos modelos fue descrita una tendencia bastante similar en ambas direcciones de estudio X y Y. Siendo los desplazamientos mucho mayores al aplicar el estudio de interacción suelo-estructura en los modelos de base rígida, lo cual indicó que a medida que la estructura poseía un ángulo elevado respecto a las líneas resistentes en dirección $Y$, y a su vez el suelo de fundación que los soporta es más flexible, los desplazamientos tenderán a incrementarse desde la base. Por otra parte, el M4, el cual posee una mayor irregularidad en su planta, arrojó movimientos torsionales tras presentar un desplazamiento considerable en la dirección X.

- Según los valores descritos anteriormente, el cortante basal para los modelos de base rígida tiende a ser 
mayor a medida que estos se cimentan en medios de fundación más flexibles. Por otra parte, al ser aplicado el estudio de interacción sueloestructura, es de notarse un aumento considerable del cortante basal de hasta un $35 \%$ en relación a los modelos de base flexible apoyados en suelos rígidos con forma espectral S1. Este aumento indica que a pesar de que la estructura está siendo evaluada en condiciones más reales, su desempeño a nivel constructivo no va adquirir una modificación en cuento al diseño de sus elementos, debido a que el mismo es estable.

- Para las formas modales, se concluyó de acuerdo a la tendencia predominante para los modelos de base rígida no ocurren desplazamientos considerables, debido a que la estructura se ve afectada con periodos de vibración muy cortos. A diferencia de los modelos con interacción, se puede observar que estos aumentan sus desplazamientos hacia los niveles superiores, gracias que en ellos participa la mayor cantidad masa, describiendo desplazamientos hacia un solo sentido sin cruzar el eje.

\section{CONCLUSIONES}

Se presentó un estudio del comportamiento dinámico en edificaciones irregulares como: distribución irregular de la masa en uno de los pisos contiguos, variación en la geometría vertical, edificaciones apoyadas en pendiente del terreno, esbeltez excesiva y sistemas no ortogonales, considerando interacción suelo-estructura. Los resultados obtenidos permitieron realizar comparaciones en las formas de vibración, las derivas de piso y el cortante basal. Se observó una modificación considerable de los desplazamientos laterales y el cortante basal en la mayor cantidad de modelos estructurales con base flexible, respecto a los modelos de base rígida.

\section{REFERENCIAS}

Alba, O. y Homero, J (2017) Determinación de los principales problemas de configuración estructural en edificaciones, que aumenten la vulnerabilidad sísmica en el Ecuador Quito: UCE

Alfaro, P., Alonso-Chaves, F. M., Fernández, C., y Gutiérrez-Alonso, G. (2013). Fundamentos conceptuales y didácticos: La tectónica de placas, teoría integradora sobre el funcionamiento del planeta. Enseñanza de las Ciencias de la Tierra, 21(2), 168180

Arellano, M., Cavallin, J., y Lanza, F. (2018). Estudio del comportamiento dinámico en edificaciones irregulares considerando interacción suelo estructura. Universidad de Carabobo, Valencia

Arteaga, S., Malavé, J., y Olival, J. (2014). Comparative of reinforced concrete structural walls design compliant to FONDONORMA 1753:2006 AND aci 318-14, Revista Ingeniería UC, 24(1), 4

Bairral, M. (2005). Debate virtual y desarrollo profesional. Una metodología para el análisis del discurso docente. Revista de Educación, $336,439-465$

Blanco, M. (2012) Criterios fundamentales para el diseño sismorresistente, Revista de la Facultad de Ingeniería Universidad Central de Venezuela, 27 (3), 71-84.

Caicedo, M. D. (2014). Períodos de vibración de las edificaciones. Revista Arquitectura e Ingeniería, 8(2), 1 
Cruz, A. B., Barra, J. E., del Castillo, R. F., y Gutiérrez, C. (2004). La calidad del suelo y sus indicadores. Revista ecosistemas, $13(2)$

COVENIN (2001) «COVENIN 1756 "Edificaciones sismorresistente,» Normas venezolanas, Caracas

Guevara, P, Teresa, L. y A. Sánchez (2005). Los sismos de enero y febrero de 2001 en El Salvador y su impacto en las iglesias del patrimonio cultural. Boletín Técnico, 43 (3), 28-57

Hernández-Castañeda, 0., y MendozaEscobedo, C. J. (2006). Durabilidad e infraestructura: retos e impacto socioeconómico. Ingeniería, investigación y tecnología, 7(1), 57-70

Lee, C., Abou, F. y López, O. (2007). Riesgo sísmico en edificaciones escolares del tipo antiguo II. Revista facultad de ingeniería de la Universidad Central de Venezuela, 22(2), 99-109

Merrit, F. S., Loftin, M. K., y Ricketts, J. T. Manual del Ingeniero Civil. (1992). Editorial Mc Grawhill, 2.

Nuria, D., y González, A. (1993). Propiedades dinámicas de edificios de la ciudad de México. 6tas. Jornadas Chilenas de Sismología e ingeniería antisísmica. Santiago, Chile, 1, 585-594

Ordaz Schroeder, M. G., Cardona Arboleda, 0. D., Salgado Gálvez, M. A., Bernal Granados, G. A., Singh, S., y Zuloaga Romero, D. (2015). Evaluación probabilista de la amenaza sísmica a nivel mundial. En Estado del arte de ingeniería sísmica en Colombia: VII
Congreso Nacional de Ingeniería Sísmica 2015, 1-10

Pintado,P., y Ortíz, P. (2013). Diseño por desempeño de estructuras metálicas de acero mediante código FEMA utilizando ETARS.Quito: ESPE

Rucks, L., García, F., Kaplán, A., Ponce de León, J., y Hill, M. (2004). Propiedades físicas del suelo. Universidad de la República, Facultad de Agronomía, Departamento de Suelos y Aguas, Montevideo, Uruguay

Tejada, J. (2005). El trabajo por competencias en el práticum; cómo organizarlo y cómo evaluarlo. Revista electrónica de Investigación educativa, $7(2), 1-31$

Vanegas Gallo, J., y Giraldo Agudelo, M. C. (2017). Evaluación de la vulnerabilidad estructural de las edificaciones indispensables del sector tres de Santa Rosa de Cabal (Tesis doctoral, Universidad Libre Seccional Pereira)

Vielma, J. C., Mulder, M. M. (2018). Factores de prestaciones sísmicas de edificios con irregularidad en planta. Monograph Series in Earthquake Engineering, editor AH Barbat

Yamin, L. E., Rodríguez, Á. E., Fonseca, L. R., Reyes, J. C., y Phillips, C. A. (2003). Comportamiento sísmico y alternativas de rehabilitación de edificaciones en adobe y tapia pisada con base en modelos a escala reducida ensayados en mesa vibratoria. Revista de ingeniería, (18), 175-192 